\title{
Efficacy of prolonged ingestion of Lactobacillus acidophilus L-92 in adult patients with atopic dermatitis
}

\author{
Kozo Yamamoto, ${ }^{* 1}$ Kazuhito Yokoyama, ${ }^{*}$ Takehisa Matsukawa, ${ }^{*}$ Sayaka Kato, $†$ Shinji Kato, $†$ \\ Kazuhisa Yamada, $\uparrow$ and Tatsuhiko Hirota† \\ *Department of Epidemiology and Environmental Health, Juntendo University Faculty of Medicine, Tokyo 113-8421, Japan \\ †Microbiology \& Fermentation Laboratory, Asahi Group Holdings Ltd., Kanagawa 252-0206, Japan
}

\begin{abstract}
To evaluate the safety and efficacy of prolonged ingestion of Lactobacillus acidophilus L-92 (L-92) on skin symptoms in adult atopic dermatitis (AD) patients, a placebo-controlled double-blinded parallel-group comparison study was performed. This included daily administration of heat-killed and dried L-92 or placebo for $24 \mathrm{wk}$ in $50 \mathrm{AD}$ patients who were $16 \mathrm{yr}$ old or older. The severity of skin symptoms was evaluated at baseline and at $4,8,12,16,20$, and 24 wk during the intervention using the investigator global assessment, eczema area and severity index, and scoring atopic dermatitis. Serum cytokine and blood marker levels were also measured at baseline and at 4, 8, 16, and $24 \mathrm{wk}$ during the intervention. No adverse events were reported during the study period. Compared with the placebo group, the L-92 group showed significant decreases in investigator global assessment, eczema area and severity index, and scoring atopic dermatitis. Subjective symptoms in adult AD patients were reduced by intake of L-92. Furthermore, it was suggested that sustained ingestion of L-92 resulted in suppression of scratching behavior and maintenance of remission status of skin symptoms. Sixteen weeks after the study commenced, a significant decrease in lactate dehydrogenase and a significant increase in transforming growth factor- $\beta$ were observed in the L-92 group compared with the placebo group. In the L-92 group, a significant elevation of IL-12 (p70) level at the end of treatment period compared with before the treatment was observed. This study suggested that L-92 suppresses type-2-helper-Tcell-dominant inflammation by activating regulatory $\mathrm{T}$ cells and type 1 helper $\mathrm{T}$ cells.
\end{abstract}

Received November 6, 2015.

Accepted March 6, 2016.

${ }^{1}$ Corresponding author: kzyamamo@juntendo.ac.jp
Key words: Lactobacillus acidophilus, atopic dermatitis, placebo-controlled double-blinded parallelgroup comparison study, allergy

\section{INTRODUCTION}

Atopic dermatitis (AD) is a chronic inflammatory skin disease with relapsing pruritic eczema, involving allergic and immunological abnormalities. Atopic dermatitis often interferes with daily activities due to symptoms of itching, scratching, and epithelial damage sustained through these activities. The cause of AD has not been fully elucidated, but it has been reported that a variety of factors, such as genetic background and collapse of skin barrier function, are important in precipitating the disease (Harris et al., 2001). One of the dominating hypotheses at this moment is that $\mathrm{AD}$ is caused by penetration of allergens in the setting of Th2-predominant conditions, which arise from an imbalance of type 1 helper T cells (Th1) and type 2 helper T cells (Th2).

Probiotics are defined as live microorganisms that confer health benefits on the host when administered in therapeutic quantities. Several studies have reported the efficacy of probiotics over the last few years, and it is thought that probiotics provide various effects through improvement of the intestinal bacterial flora. Indeed, the number of reports related to the effect on allergic diseases is rapidly increasing in the last $20 \mathrm{yr}$. With respect to allergic diseases, many reports are available on the effect of probiotics on AD. For example, Lactobacillus rhamnosus GG strain (Sistek et al., 2006) and various other strains including Lactobacillus acidophilus (Torii et al., 2011), and Lactobacillus plantarum (Yoshida et al., 2013) have been shown to have significant effects on AD. In several studies, probiotics were shown to be effective in adult patients with refractory $\mathrm{AD}$ due to protracted inflammation (Kim et al., 2014). The most common mechanism underlying the efficacy of probiotics is considered to be the hygiene hypothesis (Yazdanbakhsh et al., 2002). The hygiene 
hypothesis is a theoretical concept that suggests that exposure to bacteria and viruses in the immediate environment in childhood has a strong influence on development of allergy. Although conflicting data from several studies are conflicting, it has been definitively shown that environmental microbes have influences on the immune system of the host through fundamental studies (Matricardi et al., 2000). The Th2-dominant responses result in mast cell degranulation and release of $\operatorname{IgE}$ in patients with allergy. In contrast, probiotic lactic acid bacteria suppress the Th2 reaction by enhancing Th1 response in such patients. Furthermore, some strains of lactic acid bacteria stimulate the release of cytokines involved in inflammatory suppression, such as IL-10 and transforming growth factor- $\beta$ (TGF- $\beta$ ). These cytokines alleviate excessive inflammatory reaction by inducing regulatory T cells (Treg; Doganci et al., 2005; Smits et al., 2005; Konstantinov et al., 2008; Karimi et al., 2009). Furthermore, Konstantinov et al. (2008) reported that sufficient oral or topical administration of nonviable microbial cells (intact or broken) and crude cell extracts (i.e., with complex chemical composition) confer a benefit to the human or animal consumer (paraprobiotics). However, in humans, Boyle et al. (2008) concluded that intake of probiotics had no efficacy on AD based on data from 12 randomized controlled trials, which assessed administration of probiotics to patients with $\mathrm{AD}$. The effects of probiotics on $\mathrm{AD}$ are dependent on appropriate administration, duration, and dosage, as well as dosage of various strains. Therefore, further studies are necessary.

Lactobacillus acidophilus L-92 (L-92) is a Lactobacillus strain selected from several strains and has been shown to have potential for anti-allergic properties (Ishida et al., 2003). Efficacy of heat-killed L-92 for treatment of hay fever (Ishida et al., 2005b), perennial allergic rhinitis (Ishida et al., 2005a), and AD (Torii et al., 2011) have been reported. Furthermore, scoring atopic dermatitis (SCORAD) scores significantly improved in adult patients with $\mathrm{AD}$ when L-92 was administered over a period of $8 \mathrm{wk}$ in conjunction with their usual medication (Inoue et al., 2014). During clinical treatment, it would be useful to evaluate the efficacy of L-92 sustainably given that AD tends to follow a chronic course.

To date, few studies are available relating to the effects of long-term intake of lactic acid bacteria in conjunction with the prescribed medication in adult patients with AD. Therefore, we examined the safety and the additive protective effect of L-92 with prescribed medication in adult patients with chronic progression of $\mathrm{AD}$. We did this by evaluating variations in skin symptoms and cytokines when L-92 was administered in addition to prescribed medication over a period of 24 wk in adult patients with $\mathrm{AD}$.

\section{MATERIALS AND METHODS}

Patients who satisfied all of the following registration criteria were included in this study: (1) patients with mild to moderate AD diagnosed according to the Guideline for Management of AD by the Japanese Dermatological Association; (2) patients who were prescribed standard medication in accordance with the Guidelines for the Management of Atopic Dermatitis of the Japanese Dermatological Association; (3) patients who did not receive administration of an antibiotic within a month of the observation period; (4) patients who refrained from positive ingestion of foods containing lactic acid bacteria as well as antibiotics and antiflatulents, which may have had an effect on the intestinal flora during the study period; and (5) patients without complications of $\mathrm{AD}$ at the start of the study.

Exclusion criteria for the participation to this study were as follows: (1) patients with personal history or potential allergy against the test food; (2) patients with serious disease including diabetes, gastrointestinal disease, renal disease, and heart disease; (3) patients who could not ingest tablets on a regular response; and (4) other patients who were determined as excludable by a clinical investigator.

This study was implemented with consideration for the medical ethics in accordance with the spirit of the Declaration of Helsinki (adopted in 1964, amended in 2008). The ethics committee of Juntendo University approved this study on June 3, 2013.

Fifty-seven patients were included in this study; patients had $\mathrm{AD}$, were $16 \mathrm{yr}$ or older, and received outpatient care at the International University of Health and Welfare, Shioya Hospital (Tochigi, Japan), or Kawasakinanbu Hospital (Kanagawa, Japan). Incident cases were not included. All subjects provided written consent. The subjects were assigned by an envelope method; 28 and 29 patients were assigned to the L-92 group and placebo group, respectively.

All subjects received standard prescribed medication appropriate to the severity of symptoms of the disease, including moisture retention using heparin analog and application of external steroid drugs, in accordance with the Guidelines for the Management of Atopic Dermatitis of the Japanese Dermatological Association. The ethics committee of Juntendo University approved this study on June 3, 2013. 


\section{Test Samples}

Tablets containing $20.7 \mathrm{mg}$ of heat-killed and dried L-92 were prepared (L-92). A placebo tablet containing no L-92 with the same shape, flavor, and appearance as the L-92 tablet was also prepared. Maltose, starch, and vegetable oil were used to shape these tablets. The L-92 tablets or placebo tablets were provided to the subjects in a double-blind manner, and the subjects were asked to take the tablets every day for a period of $24 \mathrm{wk}$.

\section{Assessment of Clinical Conditions}

Severity of AD was evaluated using the investigator global assessment (IGA), eczema area and severity index (EASI), and SCORAD scores in all subjects before the intervention and at 4, 8, 12, 16, 20, and $24 \mathrm{wk}$. The IGA uses a 6 -point $(0-5)$ scale by the investigator. The EASI is a composition of scores based on the area of eczema and the intensity $(0=$ mild to $3=$ severe $)$ for 4 separate $\mathrm{AD}$ symptoms. The SCORAD is a clinical tool for assessing the severity (i.e., extent, intensity) of $\mathrm{AD}$ and subjective signs (i.e., scratching and sleeping behavior). Furthermore, blood was collected before the intervention and at 4, 8, 16, and 24 wk after the start of ingestion, and the eosinophil count, serum thymusand activation-regulated chemokine (TARC), lactate dehydrogenase (LDH), and total IgE were examined. Eosinophils were measured by the flow cytometry method, TARC and IgE were measured by the ELISA method, LDH was measured by the Japan Society of Clinical Chemistry reference method.

\section{Measurement of Cytokines}

Several cytokines were measured before the intervention and at 4, 8, 16, and 24 wk after the start of ingestion by the same blood sample. Venous blood samples $(5-10 \mathrm{~mL})$ were collected from subjects under aseptic conditions. The collected samples were immediately centrifuged to obtain serum samples. The obtained serum samples were frozen and stored at $-20^{\circ} \mathrm{C}$ until subsequent measurement.

The Bio-Plex Pro Human Cytokine Assay, 17-plex Panel (M5000031YV, Bio-Rad, Hercules, CA) was used for measurement of cytokine levels to evaluate the concentration of cytokines including IL-7, IL-8, monocyte chemotactic protein-1 (MCP-1), macrophage inflammatory protein-1 $\beta$ (MIP-1 $\beta$ ), and tumor necrosis factor- $\alpha$ (TNF- $\alpha)$. The IL-12 (p70) and TGF- $\beta$ were measured by ELISA using a human IL-12 (p70) ELISA kit (BD Biosciences, San Jose, CA) and LEGEND Free
Active TGF- $\beta 1$ kit (BioLegend, San Diego, CA), respectively.

\section{Statistical Analysis}

The Wilcoxon test was implemented for comparisons of skin symptoms (IGA, EASI, and SCORAD), measured blood test values, and serum cytokine levels at each time point of observation from 4 to 24 wk after the start of ingestion with values before the intervention. In addition, the Mann-Whitney $\mathrm{U}$ test was used to test variations ( $\Delta$ measured values) at each time point for the values before the intervention with a significance level of $P<0.05$. All statistical analyses were conducted using SPSS version 20.0 J (SPSS Inc., Chicago, IL).

\section{RESULTS}

No significant differences were observed for age, sex, and IGA score between each group, before the start of the study. Initially, 57 patients were included in the study. Seven subjects were excluded (the remainder being 22 males and 28 females). The reasons for exclusion were antibiotic intake (1 patient from the active group), nonattendance at scheduled visits (1 patient from the placebo group and 1 patient from the active group), and physician disqualification of 4 individuals for reasons unrelated to the test samples (2 patients from the placebo group and 2 patients from the active group) but who had discontinued the medication. No adverse events were observed in groups given the L-92 tablet or placebo during the study period (Table 1 ).

\section{Comparison of Changes in Skin Symptom Scores in L-92 and Placebo Groups}

Improvements were observed in all IGA, EASI, and SCORAD scores in the L-92 group compared with the placebo group. The IGA, EASI, and SCORAD scores of the L-92 group were significantly lower at wk 16, 24, and 8 after the start of ingestion compared with the placebo group, respectively (Table 2). At first, statistically significant differences between the L-92 group and placebo group were observed at wk 8 and afterward when we used SCORAD. When we used IGA and EASI, we observed statistically significant differences after wk 16 and at wk 24, respectively.

For changes in blood markers, serum total $\operatorname{IgE}$ and eosinophil count were significantly decreased in both the L-92 and placebo groups. The LDH of the L-92 group was significantly decreased at wk 16 compared with the placebo group $(P=0.049$; Table 3$)$. 
Table 1. Background of subjects ${ }^{1}$

\begin{tabular}{|c|c|c|c|}
\hline Item & L-92 & Placebo & $P$-value \\
\hline Age (yr) & $25.5(16$ to 46$)$ & $27(16$ to 49$)$ & 0.82 \\
\hline IGA & 3 (2 to 4$)$ & $3(1$ to 4$)$ & 0.43 \\
\hline SCORAD & $27.75(10.20$ to 66.70$)$ & $30.05(12.30$ to 50.30$)$ & 0.94 \\
\hline TARC $(\mathrm{pg} / \mathrm{mL})$ & $195.50(125.00$ to $2,512.00)$ & $230.50(125.00$ to 906.00$)$ & 0.86 \\
\hline Total IgE (IU/mL) & $250.00(16.10$ to $12,700.00)$ & $483.50(5.00$ to $10,200.00)$ & 0.73 \\
\hline IL-8 (pg/mL) & $5.38(2.01$ to 22.78$)$ & $5.13(2.85$ to 16.34$)$ & 0.86 \\
\hline IL-12 (p70) (pg/mL) & $26.69(1.62$ to 111.14$)$ & $35.38(2.15$ to 214.87$)$ & 0.73 \\
\hline $\mathrm{MCP}-1(\mathrm{pg} / \mathrm{mL})$ & $28.25(9.21$ to 45.40$)$ & $28.86(19.20$ to 52.74$)$ & 0.56 \\
\hline MIP-1 $\beta(\mathrm{pg} / \mathrm{mL})$ & $96.89(65.50$ to 187.18$)$ & $115.19(32.60$ to 220.24$)$ & 0.92 \\
\hline TNF- $\alpha(\mathrm{pg} / \mathrm{mL})$ & $3.72(0.38$ to 14.60$)$ & 3.74 (1.04 to 23.32$)$ & 0.12 \\
\hline TGF- $\beta(\mathrm{pg} / \mathrm{mL})$ & 15.01 (2.12 to 39.78$)$ & $13.54(3.21$ to 143.25$)$ & 0.94 \\
\hline
\end{tabular}

${ }^{1}$ Values are presented as median (minimum to maximum). L-92 = Lactobacillus acidophilus L-92; IGA = investigator global assessment; EASI $=$ eczema area and severity index; SCORAD = scoring atopic dermatitis; TARC $=$ thymus- and activation-regulated chemokine.

\section{Comparison of Changes in Serum Cytokines}

At each time point of measurement, except for some samples that were unmeasurable due to the condition of the sample, the concentrations of IL-7, IL-8, MCP-1, MIP-1 $\beta$, TNF- $\alpha$, IL-12 (p70), and TGF- $\beta$ were compared with the concentration at wk 0 . A decrease in TGF- $\beta$ was observed in the placebo group at wk 16 after ingestion. For variations from wk $0(\Delta \mathrm{TGF}-\beta)$ at each time point of measurement, the serum TGF- $\beta$ level of the L-92 group at wk 16 was significantly elevated compared with the placebo group $(P=0.03$; Table 4, Figure 1).

Furthermore, a significant increase in IL-12 (p70) was observed in the L-92 group at wk $24(P=0.049$; Figure 2).

Table 2. Changes in skin symptom score ${ }^{1}$

\begin{tabular}{|c|c|c|c|}
\hline Item & L-92 & Placebo & $P$-value \\
\hline \multicolumn{4}{|l|}{$4 \mathrm{wk}$} \\
\hline IGA & $0(-1$ to 0$)$ & $0(-3$ to 0$)$ & 0.77 \\
\hline EASI & $-1.85(-11.00$ to 9.60$)$ & $-1.30(-10.60$ to 11.60$)$ & 0.95 \\
\hline SCORAD & $-2.60(-19.5$ to 25.90$)$ & $-3.60(-28.1$ to 16.80$)$ & 0.74 \\
\hline \multicolumn{4}{|c|}{ 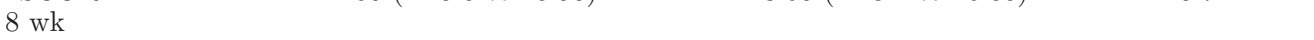 } \\
\hline IGA & $0(-1$ to 0$)$ & $0(-1$ to 1$)$ & 0.22 \\
\hline EASI & $-2.90(-13$ to 9.00$)$ & $-0.40(-12.90$ to 5.00$)$ & 0.05 \\
\hline SCORAD & $-4.90(-20.8$ to 17.80$)$ & $-0.30(-16.30$ to 14.20$)$ & $0.02 *$ \\
\hline \multicolumn{4}{|l|}{$12 \mathrm{wk}$} \\
\hline IGA & $0(-1$ to 0$)$ & $0(-1$ to 2$)$ & 0.10 \\
\hline EASI & $-3.65(-15.80$ to 8.80$)$ & $-0.70(-12.60$ to 6.70$)$ & 0.09 \\
\hline SCORAD & $-7.25(-19.70$ to 20.20$)$ & $-0.90(-18.20$ to 19.50$)$ & $0.01^{*}$ \\
\hline \multicolumn{4}{|c|}{1.20 ( 10.100020 .20$)$} \\
\hline IGA & $-1(-2$ to 1$)$ & $0(-1$ to 1$)$ & $0.03^{*}$ \\
\hline EASI & $-4.15(-18.60$ to 7.80$)$ & $-1.50(-14.60$ to 3.90$)$ & 0.16 \\
\hline SCORAD & $-8.50(-25.30$ to 19.50$)$ & $-2.00(-16.40$ to 14.40$)$ & $0.02 *$ \\
\hline \multicolumn{4}{|l|}{$20 \mathrm{wk}$} \\
\hline IGA & $-1(-3$ to 0$)$ & $0(-1$ to 1$)$ & $0.03^{*}$ \\
\hline EASI & $-5.30(-19.00$ to 7.70$)$ & $-1.50(-19.90$ to 7.30$)$ & 0.08 \\
\hline SCORAD & $-9.45(-24.70$ to 16.60$)$ & $-1.60(-17.50$ to 17.30$)$ & $0.01^{*}$ \\
\hline \multicolumn{4}{|l|}{$24 \mathrm{wk}$} \\
\hline IGA & $-1(-2$ to 0$)$ & $0(-2$ to 1$)$ & $0.0004^{* * *}$ \\
\hline EASI & $-10.65(-23.50$ to 6.90$)$ & $-0.15(-12.30$ to 12.10$)$ & $0.01^{*}$ \\
\hline SCORAD & $-6.30(-19.20$ to 4.30$)$ & $-1.10(-16.60$ to 5.60$)$ & $0.00003^{* * *}$ \\
\hline
\end{tabular}

${ }^{1}$ Scores represent changes in values from wk 0 . Values are presented as median (minimum to maximum). L-92 = Lactobacillus acidophilus L-92; IGA = investigator global assessment; EASI = eczema area and severity index; SCORAD = scoring atopic dermatitis.

${ }^{*} P<0.05,{ }^{* *} P<0.01,{ }^{* * *} P<0.001 ;$ Mann-Whitney $\mathrm{U}$ test. 
Table 3. Changes in blood test values ${ }^{1}$

\begin{tabular}{|c|c|c|c|}
\hline Item & L-92 & Placebo & $P$-value \\
\hline \multicolumn{4}{|l|}{$4 \mathrm{wk}$} \\
\hline TARC $(\mathrm{pg} / \mathrm{mL})$ & $5.00(-2,006.00$ to 190.00$)$ & $0.00(-508.00$ to 355.00$)$ & 0.90 \\
\hline Total IgE (IU/mL) & $-0.60(-640.00$ to $1,100.00)$ & $-3.00(-660.00$ to $2,400.00)$ & 0.30 \\
\hline Eosinophils $(/ \mu \mathrm{L})$ & $3.50(-504.00$ to 308.00$)$ & $-9.00(-422.00$ to 381.00$)$ & 0.73 \\
\hline $\mathrm{LDH}(\mathrm{IU} / \mathrm{mL})$ & $-12.50(-58.00$ to 25.00$)$ & $-4.00(-37.00$ to 80.00$)$ & 0.34 \\
\hline \multicolumn{4}{|l|}{$8 \mathrm{wk}$} \\
\hline TARC $(\mathrm{pg} / \mathrm{mL})$ & $5.00(-1,342.00$ to 532.00$)$ & $4.00(-479.00$ to 423.00$)$ & 0.83 \\
\hline Total IgE $(\mathrm{IU} / \mathrm{mL})$ & $-5.80(-1,390.00$ to $1,100.00)$ & $-8.60(-1,710.00$ to 800.00$)$ & 0.94 \\
\hline Eosinophils $(/ \mu \mathrm{L})$ & $-33.00(-670.00$ to 267.00$)$ & $-34.00(-345.60$ to 474.00$)$ & 0.63 \\
\hline LDH (IU/mL) & $0.50(-85.00$ to 31.00$)$ & $3.50(-37.00$ to 39.00$)$ & 0.26 \\
\hline \multicolumn{4}{|l|}{$16 \mathrm{wk}$} \\
\hline TARC $(\mathrm{pg} / \mathrm{mL})$ & $-5.00(-670.00$ to $3,650.00)$ & $-8.50(-628.00$ to 518.00$)$ & 0.71 \\
\hline Total IgE (IU/mL) & $-33.00(-3,020.00$ to $2,000.00)$ & $-65.00(-2,710.00$ to 280.00$)$ & 0.68 \\
\hline Eosinophils $(/ \mu \mathrm{L})$ & $-29.00(-599.00$ to 223.00$)$ & $-69.00(-614.00$ to 133.00$)$ & 0.27 \\
\hline $\mathrm{LDH}(\mathrm{IU} / \mathrm{mL})$ & $-5.50(-80.00$ to 77.00$)$ & $4.50(-24.00$ to 36.00$)$ & $0.049^{*}$ \\
\hline \multicolumn{4}{|l|}{$24 \mathrm{wk}$} \\
\hline TARC $(\mathrm{pg} / \mathrm{mL})$ & $0.00(-547.00$ to $2,150.00)$ & $-4.00(-442.00$ to 522.00$)$ & 0.66 \\
\hline Total IgE (IU/mL) & $-47.00(-2,400.00$ to 620.00$)$ & $-108.50(-2,090.00$ to $1,330.00)$ & 0.63 \\
\hline Eosinophils $(/ \mu \mathrm{L})$ & $-88.50(-784.00$ to 833.00$)$ & $-67.50(-722.00$ to 79.00$)$ & 0.78 \\
\hline $\mathrm{LDH}(\mathrm{IU} / \mathrm{mL})$ & $-11.50(-94.00$ to 46.00$)$ & $-1.50(-34.00$ to 53.00$)$ & 0.13 \\
\hline
\end{tabular}

${ }^{1}$ Scores represent changes in values from wk 0 . Values are presented as median (minimum to maximum). L-92 = Lactobacillus acidophilus L-92; TARC = thymus- and activation-regulated chemokine; LDH = lactate dehydrogenase.

${ }^{*} P<0.05 ;$ Mann-Whitney U test.

\section{DISCUSSION}

The effects of L-92 ingestion over a period of 6 mo in addition to prescribed medications for adult patients with $\mathrm{AD}$ was examined in this study. Three scoring methods were used for assessment of skin symptoms (Schmitt et al., 2007; Schram et al., 2012). A variety of scoring methods for skin symptoms have been used previously. Of all these methods, SCORAD is the most widely used method in Japan. This score calculates the condition of dermatitis as the sum of the severity score rated by an evaluator and the score of subjective symp- toms rated by the diseased subject. In contrast, EASI is often used for the assessment of skin conditions in Europe and the United States. However, the condition of skin rash is rated by an independent evaluator and subjective symptoms of the patient are not taken into account. For IGA, an evaluator scores the condition of dermatitis in a subject based on a holistic impression of the body (Eichenfield et al., 2002). The IGA is considered to be similar to a general practice evaluation when a physician determines the courses of treatment based on assessment of overall severity of the patient's dermatitis.

Table 4. Changes in serum cytokine level before administration in Lactobacillus acidophilus L-92 (L-92) and placebo groups $(\mathrm{pg} / \mathrm{mL})^{1}$

\begin{tabular}{lccc}
\hline Item & L-92 & Placebo & $P$-value \\
\hline IL-7 $(\mathrm{pg} / \mathrm{mL})$ & $-1.23(-5.61$ to 8.52$)$ & $-0.70(-8.75$ to 38.37$)$ & 0.53 \\
IL-8 $(\mathrm{pg} / \mathrm{mL})$ & $-1.63(-5.46$ to 6.82$)$ & $-0.86(-8.35$ to 6.30$)$ & 0.88 \\
IL-12 $(\mathrm{p} 70)(\mathrm{pg} / \mathrm{mL})$ & $11.21(-79.00$ to 69.92$)$ & $1.31(-129.81$ to 53.82$)$ & 0.29 \\
MCP-1 $(\mathrm{pg} / \mathrm{mL})$ & $16.14(-32.54$ to 17.14$)$ & $-16.20(-39.16$ to 5.39$)$ & 0.43 \\
MIP-1 $(\mathrm{pg} / \mathrm{mL})$ & $36.33(-61.04$ to 124.73$)$ & $48.23(-96.17$ to 153.25$)$ & 0.21 \\
TNF- $\alpha(\mathrm{pg} / \mathrm{mL})$ & $-2.64(-10.31$ to 3.89$)$ & $-2.87(-12.86$ to 48.01$)$ & 0.86 \\
TGF- $\beta(\mathrm{pg} / \mathrm{mL})$ & $9.73(-38.29$ to 91.21$)$ & $-5.70(-58.84$ to 44.76$)$ & 0.08 \\
$4 \mathrm{wk}$ & $1.92(-35.37$ to 105.07$)$ & $-1.42(-113.18$ to 111.29$)$ & 0.22 \\
$8 \mathrm{wk}$ & $2.52(-20.08$ to 84.90$)$ & $-5.21(-73.07$ to 22.61$)$ & $0.03^{*}$ \\
$16 \mathrm{wk}$ & $-1.51(-29.63$ to 85.85$)$ & $-0.56(-90.44$ to 58.77$)$ & 0.91 \\
$24 \mathrm{wk}$ & &
\end{tabular}

${ }^{1}$ Values represent changes in values from wk 0 . Values are presented as median (minimum to maximum). MCP $=$ monocyte chemotactic protein; $\mathrm{MIP}=$ macrophage inflammatory protein; $\mathrm{TNF}=$ tumor necrosis factor; $\mathrm{TGF}=$ transforming growth factor.

${ }^{*} P<0.05$; Mann-Whitney U test. 


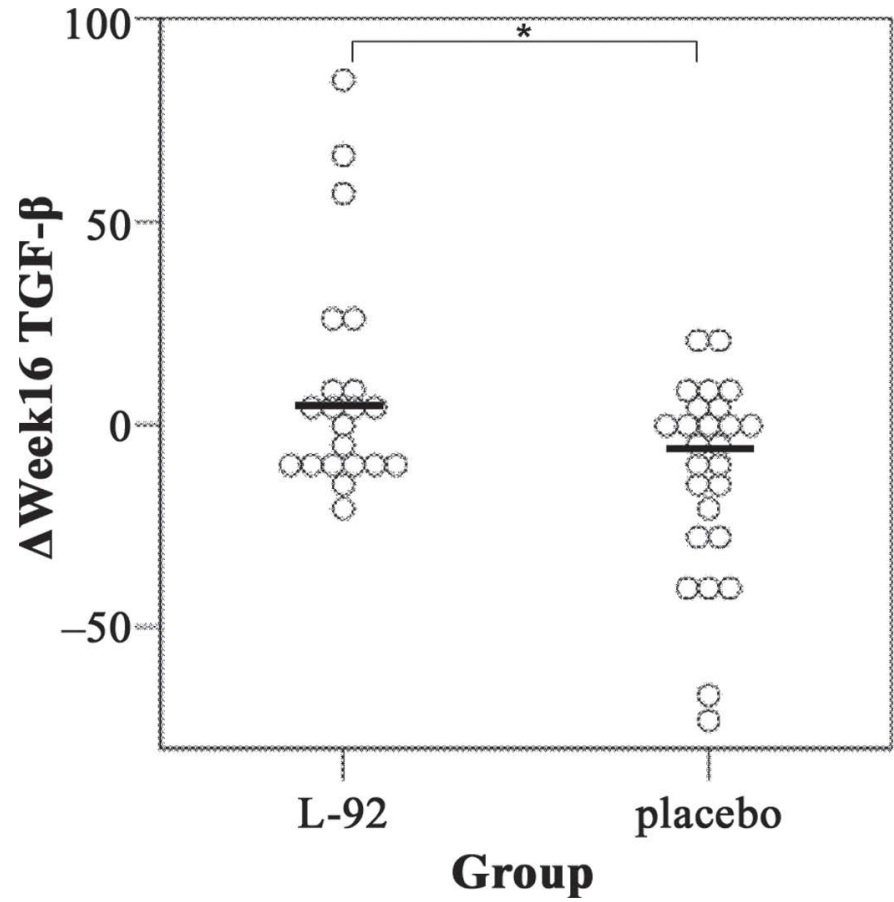

Figure 1. Changes in transforming growth factor- $\beta$ (TGF- $\beta$ ) $16 \mathrm{wk}$ after intake. Mann-Whitney U test was used for the analysis. L-92 = Lactobacillus acidophilus L-92. ${ }^{*} P<0.05$.

Of these 3 evaluation indicators, improvement was observed with SCORAD at the earliest time point in this study. Therefore, it is suggested that subjective symptoms related to itch and attendant lack of sleep were alleviated by the combined use of L-92 with prescribed medication. Significant differences in changes in IGA and EASI in the L-92 group compared with the placebo group were observed later than the change in SCORAD. Although the change in scores were small, subjective improvements were confirmed in this study of the combined use of L-92 with prescribed medication in adults with $\mathrm{AD}$.

In part, the effects of combined use of L-92 can be summarized by observing amelioration in symptoms of AD using several indicators in this study. Several evaluation indicators for the skin symptoms of $\mathrm{AD}$ have been proposed in addition to the indicators used in this study, and the number of these indicators is estimated to be more than 20 (Schmitt et al., 2007). Each indicator is calculated by the different aspects, and this may affect the result. Currently, dermatologists are engaged on the harmonizing outcome measures for eczema (HOME) initiative, which aims to define the core outcome domains to be used for clinical trials (Schmitt et al., 2015). Use of such indicators would be necessary in evaluating the additional effect of L-92 in the future.
A significant improvement was observed in the total $\operatorname{IgE}$ and eosinophil count in both L-92 and placebo groups in this study, whereas significant suppression of elevation of $\mathrm{LDH}$ was also observed at wk 16 in the L-92 group. A significant correlation between LDH and the condition of patient with $\mathrm{AD}$ has previously been reported, and $\mathrm{LDH}$ is a key measurement that determines the progress of AD (Mukai et al., 1990). It is thought that $\mathrm{LDH}$ becomes elevated as a result of inflammatory conditions induced by a collapse of the skin tissue structure due to scratching behavior. Therefore, we believe that the collapse of skin tissue was abrogated by suppression of itch in the L-92 group compared with the placebo group. As a consequence, L-92 may suppress the scratching behavior in patients with AD, which appears to be consistent with the evaluation of the aforementioned skin symptom scores.

For further analysis regarding the mechanism of action during the combination use of L-92, measurement of cytokines in the peripheral blood was performed. From these data, a significant elevation of TGF- $\beta$ was observed in the L-92 group compared with the placebo group. The TGF- $\beta$ is known as an immunosuppressive cytokine, and it has been reported that TGF- $\beta$ induces Foxp3-positive Treg in the periphery (Chen et al., 2003). The Treg cells are reported as cells capable of acting on inflammatory suppression. A previous study confirmed an increase in production of TGF- $\beta$ in the Peyer's patch in mice sensitized to ovalbumin

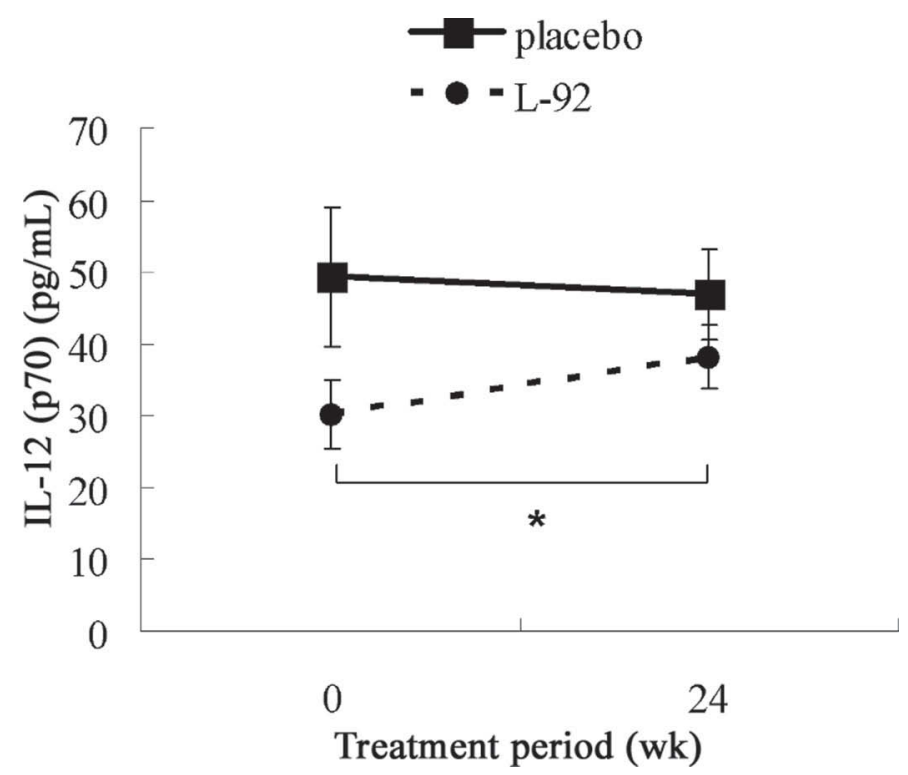

Figure 2. Changes in IL-12 (p70). Values are presented as average \pm SE. Wilcoxon signed rank test was used. L-92 = Lactobacillus acidophilus $\mathrm{L}-92 .{ }^{*} P<0.05$. 
(OVA) and given L-92 and OVA (Torii et al., 2007). Furthermore, elevation of TGF- $\beta$ in the L-92 group was also confirmed in a study conducted in adult patients with $\mathrm{AD}$, and reproducibility of this result was also confirmed in this study (Inoue et al., 2014).

In addition, a significant elevation of the IL-12 (p70) level was observed in the L-92 group compared with before and after intake of L-92. In a previous study, the production of IL-12 (p70) was increased when the L-92 strain was added to isolated and cultured spleen cells of mice sensitized to OVA at the time of antigen stimulation with OVA (Sagitani, 2010). Thus, it seems that allergic symptoms are controlled by improving the balance between Th1 and Th2 cells, through activation of Th1 cells and suppressing Th2 cells in Th2-dominant allergic diseases. Several in vitro studies have shown that lactic acid bacteria can induce Th1 cytokines (Christensen et al., 2002; Zeuthen et al., 2006). However, a significant variation in human peripheral blood has been confirmed only in a few cases (Vliagoftis et al., 2008). Additionally, the activation of Th1 and induction of TGF- $\beta$ have been confirmed. These data may appear to be conflicting. However, different cytokines are induced depending on the condition of the disease and subjects. Therefore, further analysis of the detailed immunological mechanism is required before the most suitable method of lactic acid bacteria used for treatment of $\mathrm{AD}$ is resolved.

In conclusion, in adult patients showing chronic progression of $\mathrm{AD}$, we showed that $\mathrm{AD}$ symptoms could be improved when long-term intake of the L-92 strain was combined with prescribed medications.

\section{ACKNOWLEDGMENTS}

This study was partially funded by research expenses from Microbiology \& Fermentation Laboratory, Calpis Co., Ltd. (Kanagawa, Japan). This study was performed in collaboration with the Institute for Environmental and Gender Specific Medicine, Juntendo University.

\section{REFERENCES}

Boyle, R. J., F. J. Bath-Hextall, J. Leonardi-Bee, D. F. Murrell, and M. L. Tang. 2008. Probiotics for treating eczema. Cochrane Database Syst. Rev. CD006135.

Chen, W., W. Jin, N. Hardegen, K. J. Lei, L. Li, N. Marinos, G. McGrady, and S. M. Wahl. 2003. Conversion of peripheral CD4+CD25- naive $\mathrm{T}$ cells to $\mathrm{CD} 4+\mathrm{CD} 25+$ regulatory $\mathrm{T}$ cells by TGF-beta induction of transcription factor Foxp3. J. Exp. Med. 198:1875-1886.

Christensen, H. R., H. Frokiaer, and J. J. Pestka. 2002. Lactobacilli differentially modulate expression of cytokines and maturation surface markers in murine dendritic cells. J. Immunol. 168:171-178.

Doganci, A., T. Eigenbrod, N. Krug, G. T. De Sanctis, M. Hausding, V. J. Erpenbeck, B. Haddad el, H. A. Lehr, E. Schmitt, T. Bopp,
K. J. Kallen, U. Herz, S. Schmitt, C. Luft, O. Hecht, J. M. Hohlfeld, H. Ito, N. Nishimoto, K. Yoshizaki, T. Kishimoto, S. RoseJohn, H. Renz, M. F. Neurath, P. R. Galle, and S. Finotto. 2005. The IL-6R alpha chain controls lung CD4+CD25+ Treg development and function during allergic airway inflammation in vivo. J. Clin. Invest. 115:313-325.

Eichenfield, L. F., A. W. Lucky, M. Boguniewicz, R. G. Langley, R. Cherill, K. Marshall, C. Bush, and M. Graeber. 2002. Safety and efficacy of pimecrolimus (ASM 981) cream 1\% in the treatment of mild and moderate atopic dermatitis in children and adolescents. J. Am. Acad. Dermatol. 46:495-504.

Harris, J. M., P. Cullinan, H. C. Williams, P. Mills, S. Moffat, C. White, and A. J. Newman Taylor. 2001. Environmental associations with eczema in early life. Br. J. Dermatol. 144:795-802.

Inoue, Y., T. Kambara, N. Murata, J. Komori-Yamaguchi, S. Matsukura, Y. Takahashi, Z. Ikezawa, and M. Aihara. 2014. Effects of oral administration of Lactobacillus acidophilus L-92 on the symptoms and serum cytokines of atopic dermatitis in Japanese adults: A double-blind, randomized, clinical trial. Int. Arch. Allergy Immunol. 165:247-254.

Ishida, Y., I. Bandou, H. Kanzato, and N. Yamamoto. 2003. Decrease in ovalbumin specific IgE of mice serum after oral uptake of lactic acid bacteria. Biosci. Biotechnol. Biochem. 67:951-957.

Ishida, Y., F. Nakamura, H. Kanzato, D. Sawada, H. Hirata, A. Nishimura, O. Kajimoto, and S. Fujiwara. 2005a. Clinical effects of Lactobacillus acidophilus strain L-92 on perennial allergic rhinitis: A double-blind, placebo-controlled study. J. Dairy Sci. 88:527-533.

Ishida, Y., F. Nakamura, H. Kanzato, D. Sawada, N. Yamamoto, H. Kagata, M. Oh-Ida, H. Takeuchi, and S. Fujiwara. 2005b. Effect of milk fermented with Lactobacillus acidophilus strain L-92 on symptoms of Japanese cedar pollen allergy: A randomized placebo-controlled trial. Biosci. Biotechnol. Biochem. 69:1652-1660.

Karimi, K., M. D. Inman, J. Bienenstock, and P. Forsythe. 2009. Lactobacillus reuteri-induced regulatory $\mathrm{T}$ cells protect against an allergic airway response in mice. Am. J. Respir. Crit. Care Med. 179:186-193

Kim, S. O., Y. M. Ah, Y. M. Yu, K. H. Choi, W. G. Shin, and J. Y. Lee. 2014. Effects of probiotics for the treatment of atopic dermatitis: A meta-analysis of randomized controlled trials. Ann. Allergy Asthma Immunol. 113:217-226.

Konstantinov, S. R., H. Smidt, W. M. de Vos, S. C. Bruijns, S. K. Singh, F. Valence, D. Molle, S. Lortal, E. Altermann, T. R. Klaenhammer, and Y. van Kooyk. 2008. S layer protein A of Lactobacillus acidophilus NCFM regulates immature dendritic cell and $\mathrm{T}$ cell functions. Proc. Natl. Acad. Sci. USA 105:19474-19479.

Matricardi, P. M., F. Rosmini, S. Riondino, M. Fortini, L. Ferrigno, M. Rapicetta, and S. Bonini. 2000. Exposure to foodborne and orofecal microbes versus airborne viruses in relation to atopy and allergic asthma: Epidemiological study. BMJ 320:412-417.

Mukai, H., T. Noguchi, K. Kamimura, K. Nishioka, and S. Nishiyama. 1990. Significance of elevated serum LDH (lactate dehydrogenase) activity in atopic dermatitis. J. Dermatol. 17:477-481.

Sagitani, A. 2010. Anti-allergic Effects of Lactobacillus acidophilus L-92 strain. Japanese Journal of Lactic Acid Bacteria 21:207-213.

Schmitt, J., C. Apfelbacher, P. I. Spuls, K. S. Thomas, E. L. Simpson, M. Furue, J. Chalmers, and H. C. Williams. 2015. The Harmonizing Outcome Measures for Eczema (HOME) roadmap: A methodological framework to develop core sets of outcome measurements in dermatology. J. Invest. Dermatol. 135:24-30.

Schmitt, J., S. Langan, and H. C. Williams. 2007. What are the best outcome measurements for atopic eczema? A systematic review. J. Allergy Clin. Immunol. 120:1389-1398.

Schram, M. E., P. I. Spuls, M. M. Leeflang, R. Lindeboom, J. D. Bos, and J. Schmitt. 2012. EASI, (objective) SCORAD and POEM for atopic eczema: Responsiveness and minimal clinically important difference. Allergy 67:99-106.

Sistek, D., R. Kelly, K. Wickens, T. Stanley, P. Fitzharris, and J. Crane. 2006. Is the effect of probiotics on atopic dermatitis confined to food sensitized children? Clin. Exp. Allergy 36:629-633.

Smits, H. H., A. Engering, D. van der Kleij, E. C. de Jong, K. Schipper, T. M. van Capel, B. A. Zaat, M. Yazdanbakhsh, E. A. Wie- 
renga, Y. van Kooyk, and M. L. Kapsenberg. 2005. Selective probiotic bacteria induce IL-10-producing regulatory $\mathrm{T}$ cells in vitro by modulating dendritic cell function through dendritic cell-specific intercellular adhesion molecule 3-grabbing nonintegrin. J. Allergy Clin. Immunol. 115:1260-1267.

Torii, A., S. Torii, S. Fujiwara, H. Tanaka, N. Inagaki, and H. Nagai. 2007. Lactobacillus acidophilus strain L-92 regulates the production of Th1 cytokine as well as Th2 cytokines. Allergol. Int. 56:293-301.

Torii, S., A. Torii, K. Itoh, A. Urisu, A. Terada, T. Fujisawa, K. Yamada, H. Suzuki, Y. Ishida, F. Nakamura, H. Kanzato, D. Sawada, A. Nonaka, M. Hatanaka, and S. Fujiwara. 2011. Effects of oral administration of Lactobacillus acidophilus L-92 on the symptoms and serum markers of atopic dermatitis in children. Int. Arch. Allergy Immunol. 154:236-245.

Vliagoftis, H., V. D. Kouranos, G. I. Betsi, and M. E. Falagas. 2008 Probiotics for the treatment of allergic rhinitis and asthma: Sys- tematic review of randomized controlled trials. Ann. Allergy Asthma Immunol. 101:570-579.

Yazdanbakhsh, M., P. G. Kremsner, and R. van Ree. 2002. Allergy, parasites, and the hygiene hypothesis. Science 296:490-494.

Yoshida, T., W. Fujiwara, M. Enomoto, S. Nakayama, H. Matsuda, H. Sugiyama, M. Shimojoh, S. Okada, and M. Hattori. 2013. An increased number of CD4+CD25+ cells induced by an oral administration of Lactobacillus plantarum NRIC0380 are involved in antiallergic activity. Int. Arch. Allergy Immunol. 162:283-289.

Zeuthen, L. H., H. R. Christensen, and H. Frokiaer. 2006. Lactic acid bacteria inducing a weak interleukin-12 and tumor necrosis factor alpha response in human dendritic cells inhibit strongly stimulating lactic acid bacteria but act synergistically with gram-negative bacteria. Clin. Vaccine Immunol. 13:365-375. 